\title{
The effect of bilayer regions on the response of epitaxial graphene devices to environmental gating
}

R. E. Hill-Pearce, V. Eless, A. Lartsev, N. A. Martin, I. L. Barker Snook, J. J. Helmore,

Rositsa Yakimova, J. C. Gallop and L. Hao

\section{Linköping University Post Print}

\section{Tweet}

N.B.: When citing this work, cite the original article.

Original Publication:

R. E. Hill-Pearce, V. Eless, A. Lartsev, N. A. Martin, I. L. Barker Snook, J. J. Helmore, Rositsa Yakimova, J. C. Gallop and L. Hao, The effect of bilayer regions on the response of epitaxial graphene devices to environmental gating, 2015, Carbon, (93), 896-902.

http://dx.doi.org/10.1016/j.carbon.2015.05.061

Copyright: Elsevier

http://www.elsevier.com/

Postprint available at: Linköping University Electronic Press

http://urn.kb.se/resolve?urn=urn:nbn:se:liu:diva-121418 


\title{
The Effect of Bilayer Regions on the Response of Epitaxial Graphene Devices to Environmental Gating
}

\author{
R. E. Hill-Pearce ${ }^{1 *}$, V. Eless ${ }^{1}$, A. Lartsev ${ }^{2}$, N. A. Martin ${ }^{1}$, I. L. Barker Snook ${ }^{1}$, J. J. Helmore ${ }^{1}$, \\ R. Yakimova ${ }^{3}$, J. C. Gallop ${ }^{1}$ and L. Hao ${ }^{1}$
}

\author{
${ }^{1}$ National Physical Laboratory, Hampton Road, Teddington, TW11 OLW, United Kingdom \\ ${ }^{2}$ Chalmers University of Technology, Göteborg, S-412 96 Sweden \\ ${ }^{3}$ Linköping University, Linköping, S-581 83 Sweden
}

Keywords: graphene, environmental gating, bilayer, scanning Kelvin probe microscopy, interface scattering

The effect of a bilayer area on the electronic response to environmental gating of a monolayer graphene Hall bar device is investigated using room temperature magnetotransport and scanning Kelvin probe microscopy measurements in a controlled environment. The device is tuned through the charge neutrality point with n-p-n- junctions formed. Scanning Kelvin probe measurements show that the work function of the monolayer graphene decreases more than that of the bilayer area however magnetotransport measurements show a larger change in carrier concentration for bilayer graphene with environmental gating. Interface scattering at the boundary between the monolayer and bilayer regions also affects device response with field-dependent suppression of the conductivity observed near the charge neutrality point. Simultaneous electronic and environmental scanning Kelvin probe measurements are used to build nano-scale maps of the work function of the device surface revealing the areas of greatest work function change with environmental gating.

\section{Introduction}

Bilayer (2LG) regions are often observed in nominally monolayer (1LG) graphene however, relatively little is understood regarding how $2 \mathrm{LG}$ patches affect device response to gating. While the production of epitaxial graphene $(\mathrm{EG})^{1}$ on silicon carbide $(\mathrm{SiC})$ with little or no 2LG growth is becoming tangible, ${ }^{2} 2 \mathrm{LG}$ islands are, at present, occasionally observed and their effect on device response warrants study. EG is often termed metrology grade graphene

*Corresponding author. E-mail: ruth.pearce@npl.co.uk Phone: 00442089437165 
due to the uniform 1LG graphene produced over the surface and the uniformity of the electronic properties, as such, EG is useful for quantum Hall metrology and has recently been used to verify the universality of the quantum Hall effect. ${ }^{3}$ At low temperatures magnetotransport measurements of EG devices ${ }^{4}$ show $2 \mathrm{LG}$ regions can be either conductive or insulating in the quantum Hall regime depending on the initial and gated carrier density. At room temperature scanning Kelvin probe microscopy (SKPM) has been used to demonstrate the different changes in the work function of $1 \mathrm{LG}$ and $2 \mathrm{LG}$ on gating due to their different band structure. ${ }^{5}$ We investigate environmental gating effects on the carrier concentration of 1LG and 2LG and the controlled formation of $p$-n- junctions on a device where 2LG is present. Tuning graphene to near the CNP enables the study of transport where electronic screening is reduced. ${ }^{6}$ For devices with a mean free path approaching the dimensions of the device the probability of scattering from phonons, defects and charged impurities is reduced and scattering at interfaces becomes more dominant, ${ }^{7}$ allowing the study of interface scattering.

Conventional back gating is challenging in EG which is not removed from the $\mathrm{SiC}$ substrate, with transfer of the graphene to other substrates altering the electronic properties ${ }^{8}$ and potentially adding structural disorder and contamination with transfer polymers. ${ }^{9}$ EG on the $\mathrm{Si}$ - face of $\mathrm{SiC}$ is electron doped due to interaction with the underlying buffer layer ${ }^{10}$ which is partially bonded to the $\mathrm{SiC}$ substrate ${ }^{11}$. Tuning of the electronic properties of EG has focused on intercalation of graphene; a dramatic reduction in sheet resistivity is observed on intercalation of electron donating $\mathrm{FeCl}_{3}$ molecules. ${ }^{12}$ Angle resolved photoemission spectroscopy of hydrogen intercalated, quasi-freestanding graphene has shown that the buffer layer becomes monolayer graphene and monolayer graphene becomes p-type bilayer graphene after intercalation treatment. ${ }^{13}$ Graphene sensor devices have been demonstrated as ultra-sensitive sensors ${ }^{14}$ with the potential to be single molecule detectors. ${ }^{15}$ This sensitivity of graphene to environmental gating makes it an attractive method of tuning the carrier concentration.

Environmental gating involves the donation and withdrawal of electrons by the controlled adsorption of gas molecules which shift the Fermi level of graphene. Environmental gating does not require a dielectric giving large effective fields as the distance between the graphene and the gate is reduced. The effective gate voltage is determined by the concentration of adsorbates and the amount of charge transferred by each adsorbed species. The effective 
charge $(q)$ or equivalent gate voltage on the graphene device can be tuned from electron doped (net positive $q$ ) through the charge neutrality point (net $q=0$ ) to hole doped (net negative $q$ ) by the adsorption of electron withdrawing gases which counteract the electron donation from the buffer layer.

Controlled environment SKPM of devices allows unparalleled access to the device surface enabling the changing work function of the device to be mapped as it is tuned through the charge neutrality point. While gated transport measurements can be used to show the overall response of the device they do not show nano-scale effects of inhomogeneity on the device response. A combination of controlled environment transport and SKPM measurements is thus useful for understanding the effect of $2 \mathrm{LG}$ inclusions and changing contact resistance for sensing applications and enable a nanoscale understanding of gating effects for all graphene devices.

We investigate graphene Hall bar devices fabricated by e- beam lithography from EG on the Si face of $4 \mathrm{H} \mathrm{SiC}$. The devices have a carrier concentrations $(n)$ of the order of $\sim 2 \times 10^{12} \mathrm{~cm}^{-2}$ in vacuum and a Hall mobility $(\mu)$ on the order of $3000 \mathrm{~V}^{-1} \mathrm{~s}^{-1} \mathrm{~cm}^{2}$ in ambient conditions at room temperature. The measured devices comprise $1 \mathrm{LG}$ and $2 \mathrm{LG}$ crosses. A Hall bar was selected with a $2 \mathrm{LG}$ region which covers the entire $2 \mathrm{LG}$ cross marked in Figure.1. The $n$ was tuned by the controlled desorption of atmospheric oxygen and water vapour and the adsorption of $\mathrm{NO}_{2}$ in $\mathrm{N}_{2}$. The devices were tuned to, and through the charge neutrality point (CNP) with the longitudinal voltages $\left(V_{x x}\right)$ between crosses and the Hall voltage $\left(V_{x y}\right)$ of each measured cross.

\section{Experimental}

Hall bar devices were defined by e-beam lithography and plasma etching of the graphene. Markers were deposited onto the graphene film and the enclosed areas were imaged using optical microscopy ${ }^{16}$ to determine the location of areas of $2 \mathrm{LG}$. Hall bars were then positioned over areas of 2LG. The devices were cleaned by contact mode atomic force microscopy (AFM) to remove any residual photo resist from the fabrication process and then annealed in vacuum to desorb gaseous adsorbates.

Hall measurements were carried out in controlled environments in field with constant applied current through the device $\left(I_{\text {channel }}\right)$. In order to correct for contact misalignment offsets in the measurements taken on the purely $1 \mathrm{LG}$ cross the Hall or transverse voltage $\left(V_{H}\right)$ values at magnetic field $B=0$ were subtracted and values were averaged for positive and negative 
applied $I_{\text {channel }}$ for each data point. The effects of contact misalignment and sample inhomogeneity were corrected for crosses with mixed 1 and 2LG by sweeping $B$ between 0 and $0.7 \mathrm{~T}$. The Hall coefficient $\left(R_{H}\right)$ is calculated from the slope of the measured $V_{H}$ for each cross. Due to the time taken to sweep $B$, each $R_{H}$ measurement point takes 3 minutes. During gas exposure the $V_{H}$ changes rapidly during each $R_{H}$ measurement, as such, the best linear fit was used to calculate $R_{H}$.

SKPM measurements were carried out on a NT-MDT environmentally controlled chamber with Bruker PFQNE Si on SiN probes. In order to calibrate the surface potential (SP) measurements it was assumed that the work function of the gold leads did not change. Changes in the measured SP of the gold leads were assumed to be due to a change in the work function of the Si tip and were subtracted from the measured SP values of the device.

\section{Results and Discussions}

\subsection{Device characterization}

The device was characterized by AFM and SKPM measurements see Figure. 1 which shows a 2LG area indicated by a brighter region on the SKPM map and outlined in blue on the topography map. The $2 \mathrm{LG}$ region covers one cross of the graphene Hall bar device. The topography map shows the step edges of the SiC substrate running at an angle of $\sim 45^{\circ}$ across the device, the Ti/Au electrodes and the edges of the device which are highlighted in Figure. 1a). For measurements of the $1 \mathrm{LG}$ cross a constant $I_{\text {channel }}$ was applied with $V_{x x}$ measured between the two $1 \mathrm{LG}$ crosses and $V_{x y}$ measured over the central 1LG cross. For whole device measurements $V_{\text {channel }}$ was monitored and $V_{x y}$ measured over the central 1LG cross and the right hand side $2 \mathrm{LG}$ cross.

a)

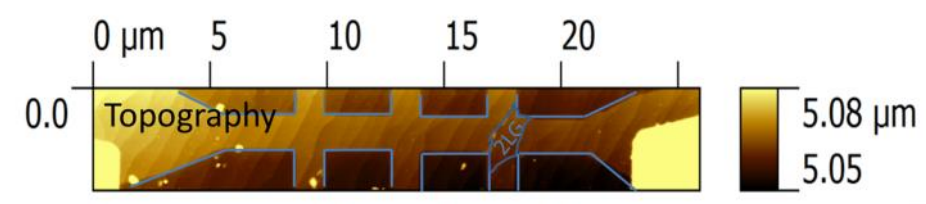

b)

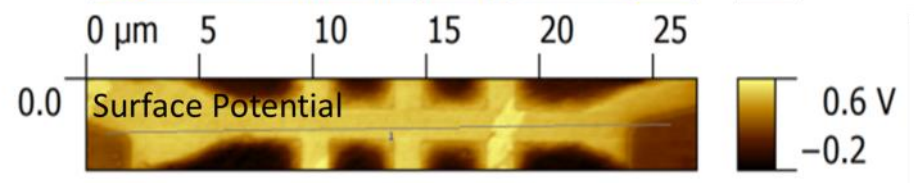

Figure 1. a) topography with outline of device and 2LG area highlighted in blue b) SKPM (unbiased) of graphene Hall bar device showing brighter areas corresponding to bilayer regions and darker areas corresponding to gold leads

\subsection{Environmental Hall measurements on $1 \mathrm{LG}$ cross}


Figure 2 shows the 1LG $R_{x x}, n$ and mobility $(\mu)$ from Hall measurements taken in increasing concentrations of $\mathrm{NO}_{2}$. The large initial response to 250 part per billion (ppb) of $\mathrm{NO}_{2}$ demonstrates the sensitivity of these devices; a 50\% change in resistance is observed in 30 minutes and a $20 \%$ increase in resistance in 5 minutes. The device demonstrates a $450 \%$ increase in resistance at the $\mathrm{CNP}$ when exposed to 42 parts per million (ppm) $\mathrm{NO}_{2}$. Increasing surface coverage of $\mathrm{NO}_{2}$ increases the equivalent positive charge $(q+)$ in the graphene counteracting the $q^{-}$from the buffer layer. When $q+=q$ - the net carrier concentration $(n),=$ 0 . For $1 \mathrm{LG}$ the $R_{x x}$ passes through a maximum as the $n$ passes through zero. The $\mu$ initially increases however, as the $n$ nears zero the $\mu$ decreases due to less screening of charge inhomogeneity and electron hole puddle formation. ${ }^{17} \mu$ passes through zero with $n$, increasing with further gating and increasing number of holes.

a)

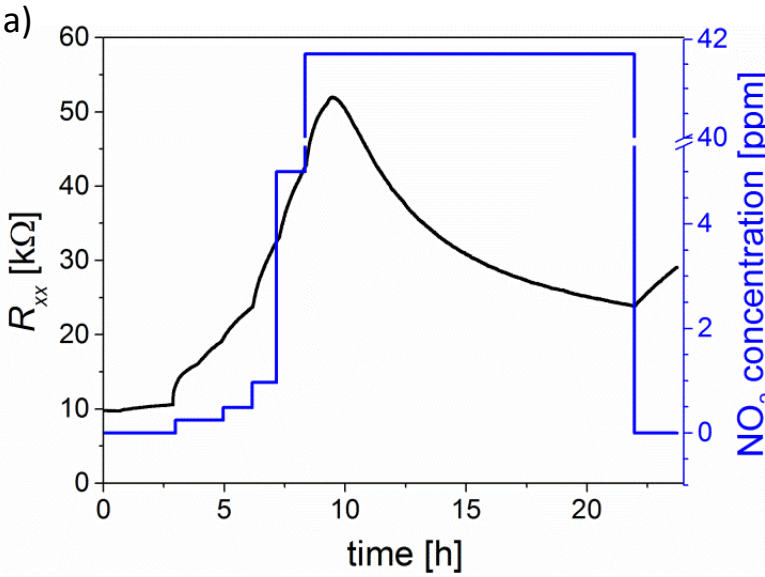

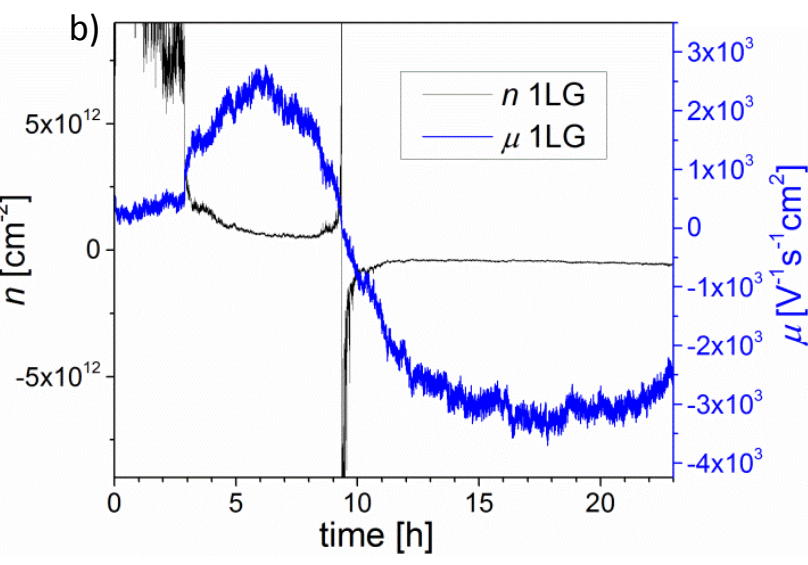

Figure 2.a) $1 \mathrm{LG} R_{x x}$ with $\mathrm{NO}_{2}$ conc. b) $1 \mathrm{LG} n$ and $\mu$, $\left(\mathrm{NO}_{2}\right.$ concentrations are as in Figure 2a)

\subsection{Mean free path of monolayer graphene}

The mean free path $(l)$ of the 1 LG was calculated using eq. (1) ${ }^{18}$ where $e$ is the elemental charge and $\hbar$ is the reduced Planck's constant

$$
l=\frac{h}{2 e} \frac{\mu}{\sqrt{\frac{n}{\pi}}}
$$

If $l<$ the channel length $\left(l_{C H}\right)$ transport through the device is expected to be diffusive. Figure 3 shows the calculated conductivity changes $(\sigma=e n \mu)$ with $\mathrm{NO}_{2}$ exposure time with values taken from data plotted in Figure, $2 \mathrm{~b}$. Figure. $3 \mathrm{~b}$ shows the relation between the calculated value of $l$ for this device and $n$. The calculated $l$ of $\sim 10 \mathrm{~nm}$ indicates that transport through the device and through the $n-p$ - junction is expected to be diffusive rather than ballistic. Mean free paths on the order of $10 \mathrm{~nm}$ have been demonstrated for EG devices ${ }^{19}$ in agreement with the data presented here. 

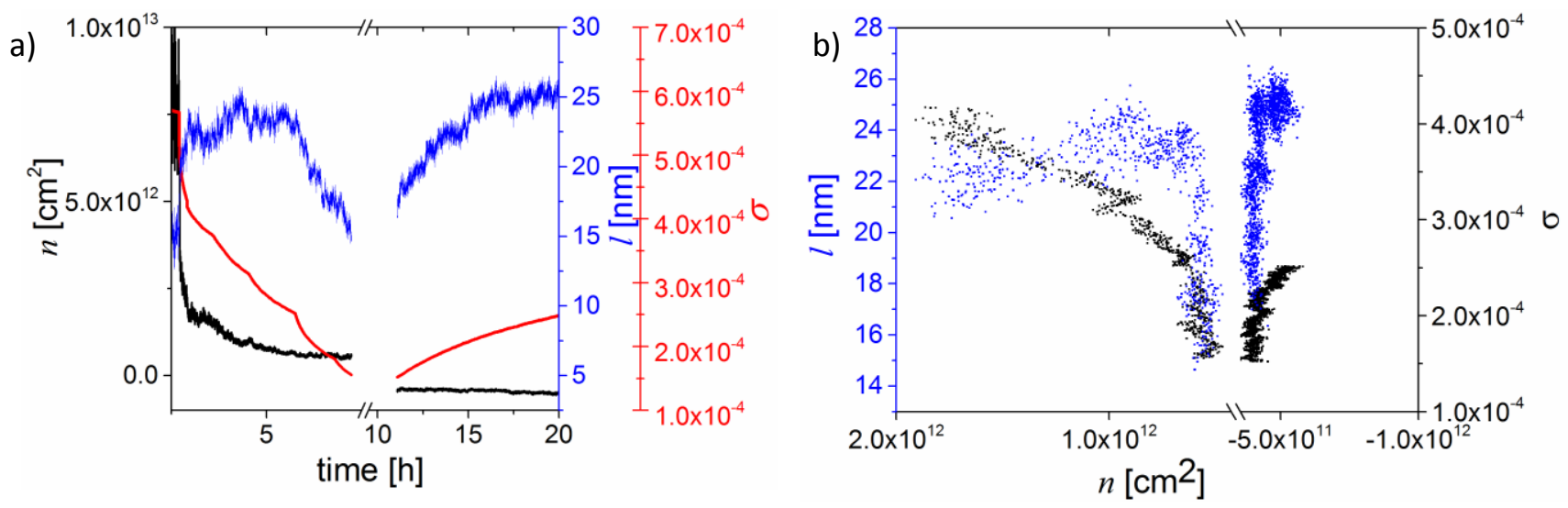

Figure 3. a) conductivity $(\sigma)$, mean free path $(l)$ and carrier concentration $(n)$ with time in $\mathrm{NO}_{2}$. b) mean free path $(l)$ and conductivity $(\sigma)$ with varying $n$. Data is not plotted for the region where divergence of $n$ is observed.

\subsection{Effect of 2LG on transport: SKPM measurements}

Measuring a 2LG inclusion on a 1LG device has the advantage that the conditions of growth and the post growth processing of the areas are identical for both regions and the difference in the response of each cross is due purely to the difference in layer thickness.

SKPM maps of the device when grounded (Figure 4b) and biased (Figure 4c.) are plotted in Figure 4 along with the topography of the device (Figure 4a). The voltage drop across the device can be visualized as a change in color gradient along the channel. Figure $4 \mathrm{~d}$ shows potential line profiles taken across the SKPM maps b) and c) from the regions marked with a blue line. The slope of the voltage drop across the channel is linear even in the region of the $2 \mathrm{LG}$ area indicating that the $2 \mathrm{LG}$ region does not affect the resistance of the channel significantly at this equivalent gating voltage.
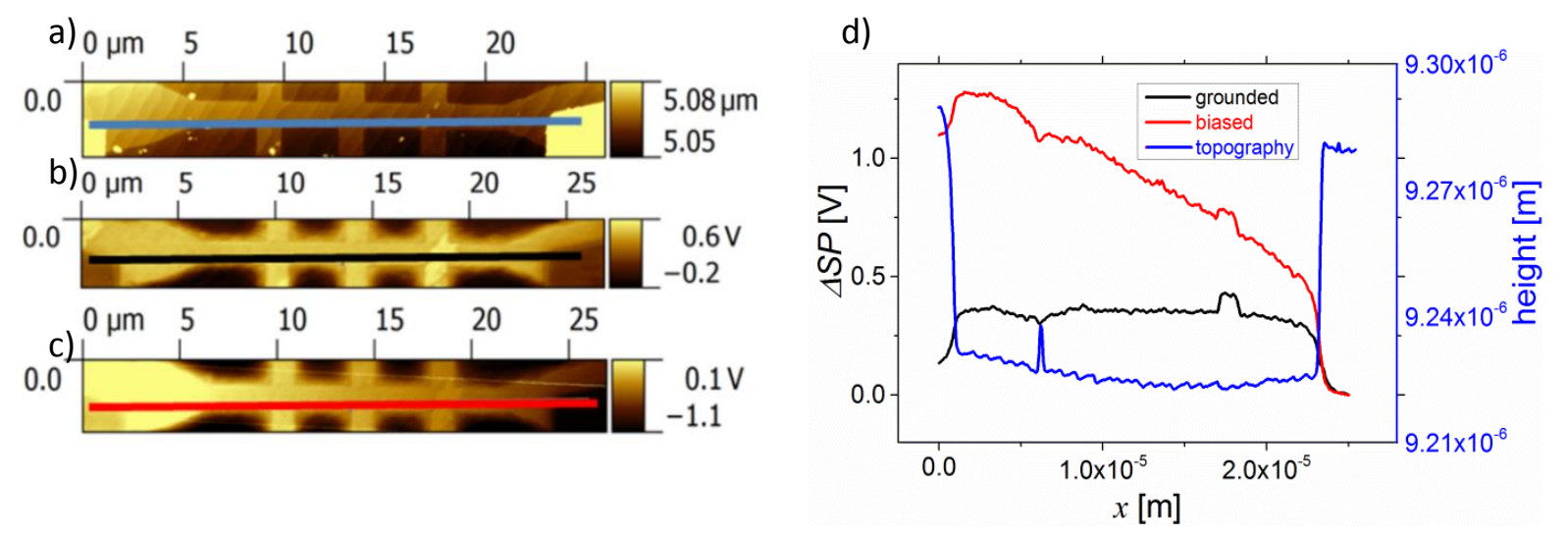

Figure 4. a) topography of device b) SKPM of grounded device c) SKPM of biased device,

d) extracted line profiles from Figures. 4. a), b) and c) 


\subsection{Effect of 2LG on transport: Hall measurements}

Transport measurements were measured at room temperature; the longitudinal and transverse voltages ( $V_{\text {channel and }} V_{H}$ respectively) were recorded. $V_{\text {channel }}$ was recorded over the channel length and $V_{H}$ was simultaneously measured over the central 1LG cross and the 2LG cross while sweeping the magnetic field between 0 and $0.7 \mathrm{~T}$ in a controlled environment. $R_{H}$ is calculated from the slope of the line of the measured $V_{H}$ with field $(B)$. For each plotted $R_{H}$ point $20 R_{\text {channel }}$ points in increasing $B$ and 20 in decreasing $B$ were measured. $R_{\text {channel }}$ is plotted as a continuous line in Figures 5a) and b). The $R_{H}$ and the calculated $n$ of the central $1 \mathrm{LG}$ cross and the $2 \mathrm{LG}$ cross are plotted as a linked scatter plot. The device resistance was monitored in a vacuum environment at $\sim 1 \times 10^{-7}$ mbar until the resistance stabilized $\sim 48$ hours. Clean, dry nitrogen (Air products, $\mathrm{BIP}^{+}$) was introduced into the chamber as a carrier gas. $1 \mathrm{ppm} \mathrm{NO}_{2}$ in $\mathrm{N}_{2}$ was used to tune the Fermi level of the device from n-type through the CNP to p-type while monitoring the $R_{H}$ and calculating $n$ for the $1 \mathrm{LG}$ and $2 \mathrm{LG}$ crosses.

a)

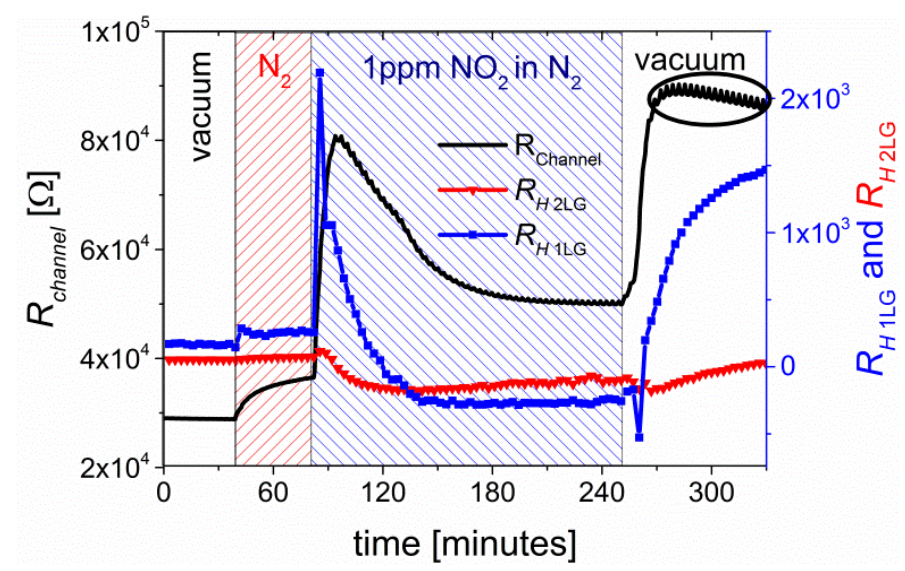

b)

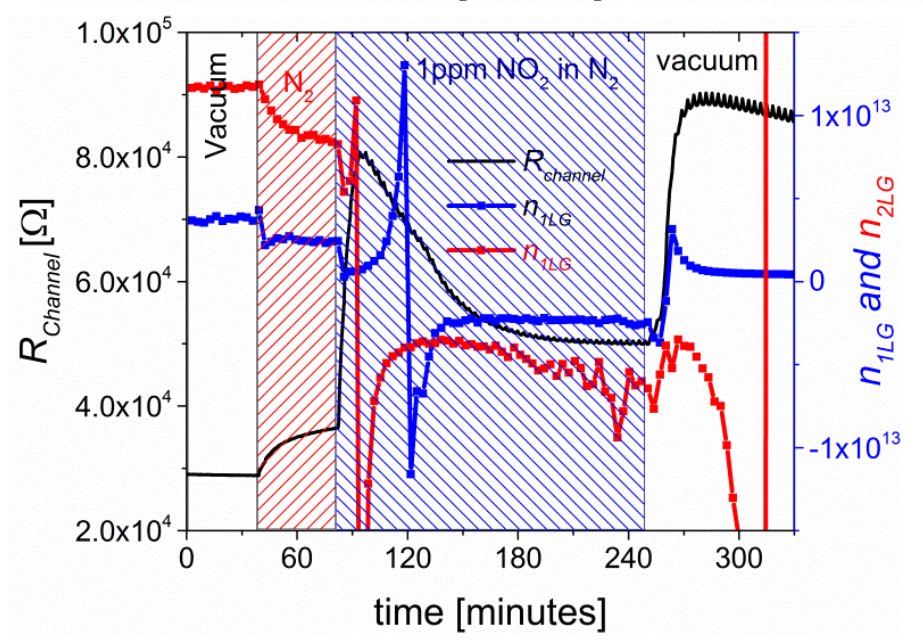

Figure 5. a) $R_{\text {channel, }} R_{H-1 \mathrm{LG} \text { and }} R_{H-2 \mathrm{LG}}$ with circled area showing suppression of conductivity with field, b) $R_{\text {channel, }} n_{1 L G}$ and $n_{2 L G}$, 
Figure 5a) shows the $R_{\text {channel }}$ and $R_{H}$ of the central $1 \mathrm{LG}$ cross and the $2 \mathrm{LG}$ cross. The resistance of the device reaches a maximum when the $2 \mathrm{LG}$ area, which dissects the channel, reaches the CNP. Figure 5b shows that the $2 \mathrm{LG}$ area reaches the CNP at a shorter time in $\mathrm{NO}_{2}$ than the $1 \mathrm{LG}$ demonstrating a larger change in $n$, but a smaller change in $R_{H}$ than the 1LG. SKPM measurements ${ }^{5}$ have shown a larger $\Delta \Phi$ for $1 \mathrm{LG}$ than $2 \mathrm{LG}$ in increasingly electron withdrawing $\mathrm{NO}_{2}$ environments due to the narrower density of states of $1 \mathrm{LG}$ compared to $2 \mathrm{LG}$.

The overall series resistance of the device is dominated by the most resistive part, with a change in the slope of $R_{x x}$ observed in Figures $5 \mathrm{a}$ and $\mathrm{b}$ when the 1LG area nears the CNP. The resistance of the device begins to stabilize as the rate of $\mathrm{NO}_{2}$ adsorption and desorption reaches equilibrium. The $R_{x x}$ increases rapidly on evacuating the chamber and desorption of $\mathrm{NO}_{2}$ until the CNP is reached. For graphene free from impurities and disorder the Fermi level is expected to lie at the Dirac point with a maximum resistance of $e^{2} / h$. ${ }^{20}$ However, the conductivity at the Dirac point is calculated to be affected by impurity scattering. The presence of disorder leads to the formation of electron and hole puddles ${ }^{17}$ which, in turn, leads to non zero conduction at the Dirac point for $1 \mathrm{LG}^{20}$ and $2 \mathrm{LG} .{ }^{21}$ Here we show that with environmental gating the maximum measured resistance of the device depends on the speed at which the gating species is adsorbed or desorbed. Different maximum resistivity values are observed when the CNP is reached by introducing $\mathrm{NO}_{2}$ and when the $\mathrm{NO}_{2}$ is slowly desorbed in vacuum.

\subsection{Field induced conductivity suppression}

Suppressed conductivity is observed along the channel with applied $B$ when the device contains an n-p-n- junction and, to a lesser extent, when one area of the device (1LG or 2LG) is close to the CNP, the conductivity change with field is highlighted in Figure 5a and also shown in figure 6). The field induced conductivity suppression is observed as one oscillation in $R_{\text {channel }}$ for each $V_{H}$ measurement point where the field is swept from 0 to $0.66 \mathrm{~T}$ and back to zero with $R_{\text {channel }}$ measured at 40 points. The effect of field on the continuous $R_{\text {channel }}$ measurements is enlarged in figure 6a. The increasing $V_{H}$ and $V_{\text {Channel }}$ with field is plotted in Figure $6 \mathrm{~b}$ when the $2 \mathrm{LG}$ is p-type and the $1 \mathrm{LG}$ is $\mathrm{n}$-type. The resistance of the device is changing rapidly at this time leading to changing values of $V_{H}$ and $V_{\text {Channel }}$ at zero field.

At the CNP screening is reduced and the effects of scattering become more pronounced. ${ }^{6}$ The observed increase in resistance with field may indicate magnetic scatterers with 
paramagnetic, vacancies and adatoms ${ }^{22}$ reported. Ferromagnetic domain walls ${ }^{23}$ and zigzag edge states have also been predicted for graphene. ${ }^{24}$ The magnetic moment of defects in graphene has been reported to be affected by doping, ${ }^{25}$ with magnetic moments near the charge neutrality point reversibly switched off by shifting the Fermi energy away from neutral. Conductivity through the device may also be affected by band gap opening which has been reported in bilayer graphene in a perpendicular magnetic field. ${ }^{26,27}$
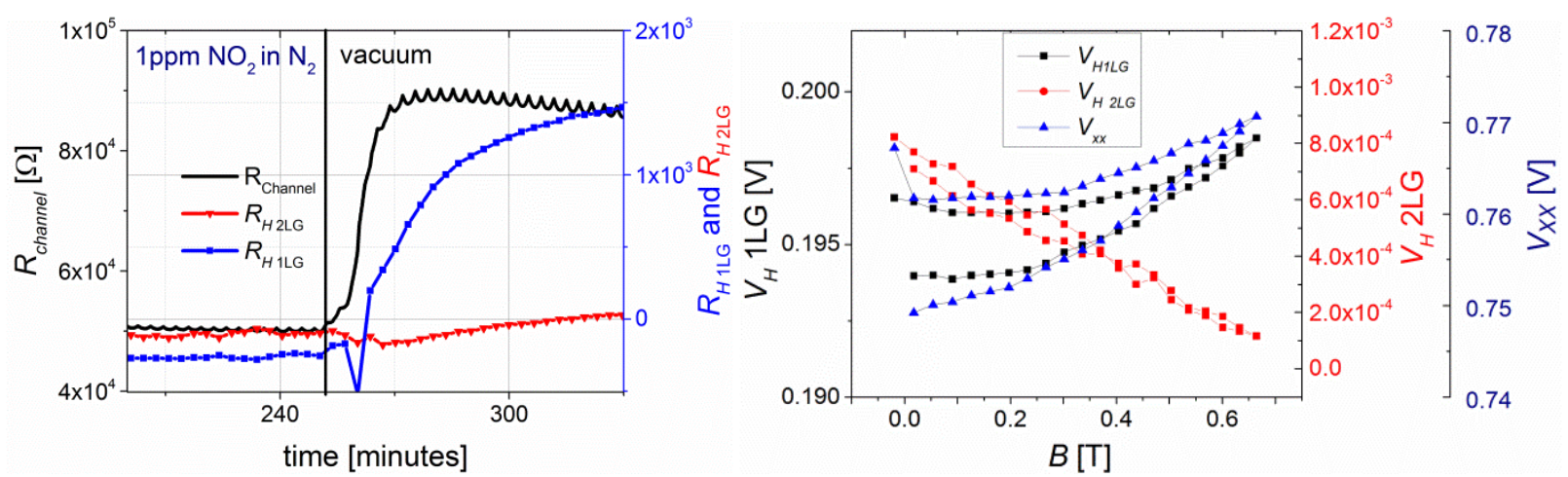

Figure 6. a) enlargement of circled region of figure 5a) b) Response of $V_{H-1 \mathrm{LG}}, V_{H-2 \mathrm{LG}}$ and $V_{x x}$ to magnetic field when the $2 \mathrm{LG}$ region is p-type and the $1 \mathrm{LG}$ region is $n$-type

Scanning potentiometry measurements ${ }^{28}$ have demonstrated local perturbations located at 12LG boundaries with first principle calculations ${ }^{28}$ showing an intrinsic 1-2LG wave function mismatch leading to localised high resistance. The doping dependant nature of these localised resonant scattering mechanisms has been proposed ${ }^{29}$. Conductive atomic force microscopy observations and first principle calculations ${ }^{29}$ indicate that this wave function mismatch gives rise to strong conduction suppression for energies within a $\pm 0.48 \mathrm{eV}$ range from the Dirac point. Within this energy range weak wave function coupling of the $1 \mathrm{LG} \pi / \pi^{*}$ bands with the first bands of the $2 \mathrm{LG}$ region is observed however, matching between the $1 \mathrm{LG} \pi / \pi^{*}$ bands and the second bands of the bilayer region is calculated to be almost ideal ${ }^{29}$.

\section{7 $\mathrm{NO}_{2}$ adsorption: effective gate voltage and $\mathrm{NO}_{2}$ density of adsorption}

The adsorption energy and electron transfer when an $\mathrm{NO}_{2}$ molecule binds to a graphene supercell has been calculated to be $\sim 0.1 e$ per molecule. ${ }^{30}$ Various configurations of oxygen up or oxygen down type adsorption give relatively similar amounts of charge transfer and similar graphene $-\mathrm{NO}_{2}$ distances, however the total amount of charge transfer may vary with supercell size. ${ }^{31}$

At the CNP the $q^{+}$from the adsorbed $\mathrm{NO}_{2}$ is equal and opposite to the $q^{-}$from the buffer layer $\left(q_{B L}=q_{N O 2}\right)$. The density of adsorbed $\mathrm{NO}_{2}$ molecules $\left(\gamma_{\mathrm{NO} 2}\right)$ required to tune the $1 \mathrm{LG}$ and 
the 2LG to the CNP is calculated along with the effective gate voltage at the CNP. The initial $n_{1 \mathrm{LG}}$ is $\sim 3 \times 10^{12} \mathrm{~cm}^{-2}$, assuming a charge transfer $(\Delta e)$ per molecule of $0.1 \mathrm{e}$ per $\mathrm{NO}_{2 \text { ads }}{ }^{30}$ gives a density of adsorbates $\gamma \mathrm{NO} 2=3 \times 10^{13} \mathrm{~cm}^{-2}$. Using theorized $\mathrm{NO}_{2}$-graphene distances $(d)$ of $3.6 \AA^{30}$ the effective gate voltage $\left(V_{e f f}\right)$ due to the transfer of charge to the $\mathrm{NO}_{2}$ molecules is calculated; $V_{\text {eff }}=\Delta \mathrm{Q} / \mathrm{C}$, where $\mathrm{C}$ is the capacitance. Assuming a value of $\varepsilon_{r}=1$ then $V_{\text {eff }}=$ $d \gamma_{\mathrm{NO} 2} \Delta e / \varepsilon_{0}$ giving a $V_{\text {eff }} 1 \mathrm{LG}=19.5 \mathrm{mV}$ and a $V_{\text {eff }} 2 \mathrm{LG}=65 \mathrm{mV}$ assuming a $n_{2 \mathrm{LG}}$ of $1 \times 10^{13} \mathrm{~cm}^{-}$ 2. For a $\mathrm{SiO}_{2}$ dielectric of $300 \mathrm{~nm}$ thickness and an $\varepsilon_{r}$ value of 3.9 the equivalent $q$ required to reach the CNP for $1 \mathrm{LG}$ would give a gate voltage of $4.17 \mathrm{~V}$, and a gate voltage of $13.91 \mathrm{~V}$ for 2LG. The larger calculated $V_{\text {eff }}$ required for tuning the $2 \mathrm{LG}$ area to the CNP and the more rapid change in $n$ of the $2 \mathrm{LG}$ suggests either that $\mathrm{NO}_{2}$ transfers more $q$ to $2 \mathrm{LG}$ than $1 \mathrm{LG}$ or that more $\mathrm{NO}_{2}$ is adsorbed on $2 \mathrm{LG}$ than $1 \mathrm{LG}$. Different chemical reactivity rates have been reported for $1 \mathrm{LG}$ and $2 \mathrm{LG}^{32,33}$

\subsection{Combined electronic and SKPM measurements}

The response of the device to $\mathrm{NO}_{2}$ was investigated with combined electrical and SKPM measurements. Figure 6 a) shows the resistance of the device with $V_{x x}$ applied along the channel and $I_{x x}$ measured along the channel in order to maintain a constant voltage drop over the device during exposure to $1 \mathrm{ppm} \mathrm{NO}_{2}$ in nitrogen. The colored numbers in Figure $6 \mathrm{a}$ indicate where scans were taken during environmental gating. The initial vacuum pressure was $\sim 1 \times 10^{-6}$ mbar, $1 \mathrm{ppm} \mathrm{NO}_{2}$ in $\mathrm{N}_{2}$ was flowed into the chamber the $\mathrm{NO}_{2}$ concentration was increased to $100 \mathrm{ppm}$ after 2 hours. The higher concentration $\mathrm{NO}_{2}$ was used as the chamber is larger ( $~ 8$ liters) than the chamber used for the Hall measurements ( $\sim 0.5$ liters). The resistance of the device increases with $\mathrm{NO}_{2}$ concentration until the CNP is reached for the 2LG area, the resistance then decreases until the chamber is evacuated. On evacuation the device resistance increases rapidly reaching a maximum at the CNP from which point the resistance decreases again. The increase in resistance on exposure to lab ambient $\left(20 \% \mathrm{O}_{2}\right.$ and $70 \% \mathrm{RH}$ ) is smaller than the increase observed for $100 \mathrm{ppm} \mathrm{NO}_{2}$ demonstrating the smaller charge transfer between $\mathrm{O}_{2}$ /water vapour and graphene than $\mathrm{NO}_{2}$ and graphene.

The SKPM maps (Figure 7b) taken at points marked on Figure 7a) show the SP change over the device. In vacuum the $2 \mathrm{LG}$ appears darker indicating a larger work function than 1LG. The change in SKPM contrast between $1 \mathrm{LG}$ and $2 \mathrm{LG}$ in different environmental conditions has been discussed ${ }^{5}$ and is assigned to the faster changing work function of $1 \mathrm{LG}$ than 2LG due to the narrower DOS of 1LG. Here we show that the change in SKPM contrast 
occurs not at the CNP but at a much higher $n$, with the contrast inversion occurring between vacuum and very low $\mathrm{NO}_{2}$ concentrations.

Figure 7c) shows extracted line profiles taken from Figure $7 \mathrm{~b}$ (marked in blue). The line profiles show the relative decrease in $\mathrm{SP}$ of the graphene with $\mathrm{NO}_{2}$ exposure. The $\mathrm{SP}$ of the 2LG remains relatively constant in vacuum, $\mathrm{N}_{2}$ and in low $\sim 1 \mathrm{ppm} \mathrm{NO}_{2}$ in $\mathrm{N}_{2}$ whereas the SP of the $1 \mathrm{LG}$ drops by $0.25 \mathrm{~V}$, the larger change in SP for $1 \mathrm{LG}$ than $2 \mathrm{LG}$ causes an inversion of the observed SKPM contrast. The contact resistance between the 1LG and the grounded Au contact pad drops with $\mathrm{NO}_{2}$ exposure as the work function of the graphene become more similar to that of the gold electrode. The difference in measured voltage drop between the graphene and the Au electrode when the device is unbiased and biased is used to calculate a contact resistance of $\sim 4 \mathrm{k} \Omega$ in vacuum. The contact resistance lowers with $\mathrm{NO}_{2}$ exposure with a $0.01 \mathrm{~V}$ drop measured in $100 \mathrm{ppm} \mathrm{NO}_{2}$ giving a resistance of $\sim 1.5 \mathrm{k} \Omega$.
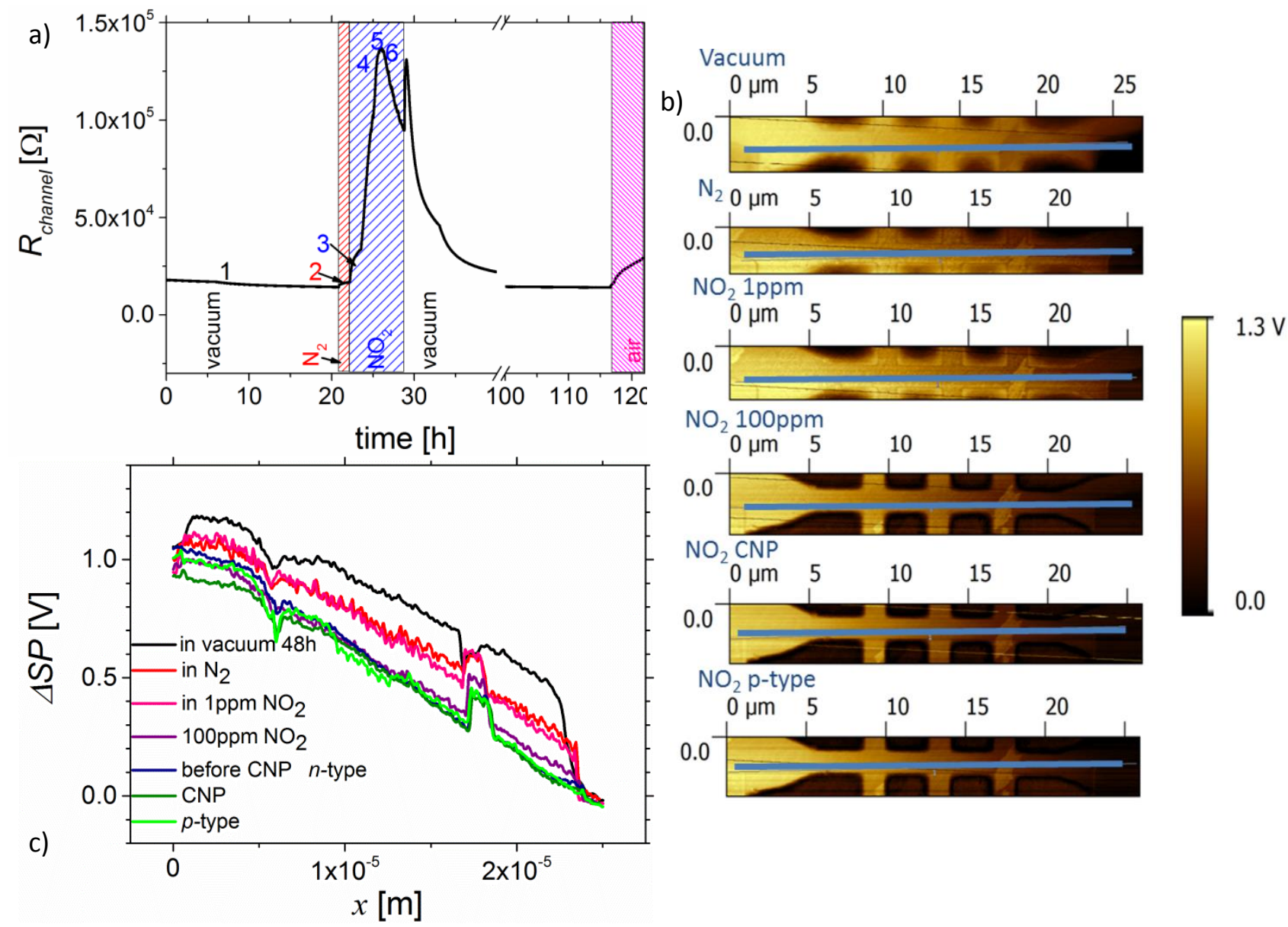

Figure 7. a) $R_{\text {channel }}$ measured during environmental SKPM, b) SKPM maps of the device taken at points 1 to 6 marked in Figures. a), c) extracted line profiles from the SKPM maps in b) showing the changing SP with environmental gating. 


\section{Conclusion}

An overview of the room temperature effects of bilayer areas on the response of graphene devices to gating has been presented. Using simultaneous transport and SKPM measurements we have demonstrated nanoscale mapping of the response of the device to gating indicating the areas of fastest change in $\Phi$ and mapping resistance as a function of device position in each environment. The effect of non-uniform response to gating in a device where $2 \mathrm{LG}$ is present is demonstrated by the formation of p-n-junctions, in this region field dependent interface scattering effects are observed. Hall measurements demonstrate the greater sensitivity of $R_{H-1 \mathrm{LG}}$ to gating than that of $R_{H-2 \mathrm{LG}}$, despite the $n$ of $2 \mathrm{LG}$ changing to a greater extent than that of $1 \mathrm{LG}$, making $1 \mathrm{LG}$ the preferred material for sensitive chemical sensors. The larger change in $n_{2 \mathrm{LG}}$ then $n_{1 \mathrm{LG}}$ indicates that gaseous species interaction with graphene may be thickness dependent.

\section{Acknowledgements}

This work was supported by the UK NMS Programme, graphene flagship and the EU EMRP project 'GraphOhm'. The EMRP is jointly funded by the EMRP the participating countries within EURAMET and the European Union. The research leading to these results has partly received funding from the European Union Seventh Framework Programme under grant agreement 604391 Graphene Flagship. We also gratefully acknowledge the funding received from the UK Department for Business, Innovation and Skills (BIS) in Sensor networks: data to knowledge, Project IRD/2013/09

\section{References}

[1] R. Yakimova, C. Virojanadara, D. Gogova, M. Syväjärvi, D. Siche, K. Larsson, L.I. Johansson, Analysis of the Formation Conditions for Large Area Epitaxial Graphene on SiC Substrates, Mater. Sci. Forum 2010, 645-648, 565-568.

[2] J. Eriksson, R. Pearce M. Andersson, T. Iakimov C. Virojanadara, M. Syväjärvi, et $a l .$, The Influence of Substrate Morphology on Thickness Uniformity and Unintentional Doping of Epitaxial Graphene on SiC, Appl. Phys. Lett. 2012, 100, 241607-241601 - 241607-241605.

[3] A. Tzalenchuk, S. Lara-Avila, A. Kalaboukhov, S. Paolillo, M. Syväjärvi, R. Yakimova, et al., Towards a Quantum Resistance Standard Based on Epitaxial Graphene, Nat. Nano. 2010, 5, 186-189. 
[4] C. Chua, M. Connolly, A. Lartsev, T. Yager, S. Lara-Avila, S. Kubatkin, S. Kopylov, et al., Quantum Hall Effect and Quantum Point Contact in Bilayer-Patched Epitaxial Graphene, Nano Lett. 2014, 14, 3369-3373.

[5] R. Pearce, J. Eriksson:, T. Iakimov, L. Hultman, A Lloyd Spetz, R. Yakimova, On the Differing Sensitivity to Chemical Gating of Single and Double Layer Epitaxial Graphene Explored Using Scanning Kelvin Probe Microscopy, ACS Nano 2013, 7, 4647-4656.

[6] T. Ohta, A. Bostwick, J. L. McChesney, T. Seyller, K. Horn, E. Rotenberg, Interlayer Interaction and Electronic Screening in Multilayer Graphene Investigated with Angle-Resolved Photoemission Spectroscopy, Phys. Rev. Lett. 2007, 98, 206802.

[7] S. Masubuchi, K. Iguchi, T. Yamaguchi, M. Onuki, M. Arai, K. Watanabe, et al., Boundary Scattering in Ballistic Graphene, Phys. Rev. Lett. 2012, 109, 036601.

[8] D. S. Lee, C. Riedl, B. Krauss, K. von Klitzing, U. Starke, J. H. Smet, Raman Spectra of Epitaxial Graphene on $\mathrm{SiC}$ and of Epitaxial Graphene Transferred to $\mathrm{SiO}_{2}$, Nano Lett. 2008, 8, 4320-4325

[9] Y.-C Lin, C. Lu, C- H- Yeh, C. Jin, K. Suenaga, P.-W Chiu, Graphene Annealing: How Clean Can It Be?, Nano Lett. 2011, 12, 414-419.

[10] S. Kopylov, A. Tzalenchuk, S. Kubatkin, V. I. Fal'ko, Charge Transfer between Epitaxial Graphene and Silicon Carbide, Appl. Phys. Lett. 2010, 97, 112109-112103

[11] C. Riedl, U. Starke, J. Bernhardt, M. Franke, K. Heinz, Structural Properties of the Graphene-SiC(0001) Interface as a Key for the Preparation of Homogeneous LargeTerrace Graphene Surfaces, Phys. Rev. B, 2007, 76, 245406.

[12] Bointon, T. H. Khrapach, I. Yakimova, R. Shytov, A. V. Craciun, M. F. Russo, S., Approaching Magnetic Ordering in Graphene Materials by $\mathrm{FeCl}_{3}$ Intercalation. Nano Letters 2014, 14, 1751-1755.

[13] C. Riedl, C. Coletti, U. Starke, Structural and Electronic Properties of Epitaxial Graphene on SiC (0001): A Review of Growth, Characterization, Transfer Doping and Hydrogen Intercalation, J. Phys. D: Appl. Phys. 2010, 43, 374009-374017.

[14] R. Pearce, T. Iakimov, M. Andersson, L. Hultman, A. Lloyd Spetz, R. Yakimova, Epitaxially Grown Graphene Based Gas Sensors for Ultra Sensitive $\mathrm{NO}_{2}$ Detection, Sensors Actuators B: Chem. 2011, 155, 451-455.

[15] F. Schedin, A. K. Geim S. V. Morozov, E. W. Hill, P. Blake, M. I. Katsnelson, et al., Detection of Individual Gas Molecules Adsorbed on Graphene, Nat. Mater. 2007, 6, 652-655. 
[16] T. Yager, A. Lartsev, S. Mahashabde, S. Charpentier, D. Davidovikj, A. Danilov, et al ., Express Optical Analysis of Epitaxial Graphene on SiC: Impact of Morphology on Quantum Transport, Nano Lett. 2013, 13, 4217-4223.

[17] J. Martin, N. Akerman, G. Ulbricht, T. Lohmann, J. H. Smet, K. von Klitzing, et al., Observation of Electron-Hole Puddles in Graphene Using a Scanning Single-Electron Transistor, Nat. Phys. 2008, 4, 144 - 148

[18] V. V. Cheianov, V. I. Fal'ko, Selective Transmission of Dirac Electrons and Ballistic Magnetoresistance of N-P Junctions in Graphene, Phys. Rev. B 2006, 74, 041403

[19] S. Lara-Avila, A. Tzalenchuk, S. Kubatkin, R. Yakimova, T. J. B. M Janssen, K. Cedergren, et al., Disordered Fermi Liquid in Epitaxial Graphene from Quantum Transport Measurements, Phys. Rev. Lett., 2011, 107, 166602.

[20] E. H. Hwang, S. Adam, S. Das Sarma, Carrier Transport in Two-Dimensional Graphene Layers, Phys. Rev. Lett. 2007, 98, 186806

[21] S. Das Sarma, E. H. Hwang, E. Rossi, Theory of Carrier Transport in Bilayer Graphene, Phys. Rev. B, 2010, 81, 161407

[22] R. R. Nair, M. Sepioni, I. Ling-Tsai, O. Lehtinen, J. Keinonen, A. V. Krasheninnikov, et al., Spin-half paramagnetism in graphene induced by point defects, Nat. Phys., 2012, 8, 199-202.

[23] M. Homma, C. Itoi, Ferromagnetic Domain Wall and Spiral Ground States in OneDimensional Deformed Flat-Band Hubbard Model, J. Stat. Phys, 2004, 117, 477-519.

[24] S. S. Alexandre, A. D. Lúcio, A. H. C. Neto, R. W. Nunes, Correlated Magnetic States in Extended One-Dimensional Defects in Graphene, Nano lett. 2012, 12, 50975102.

[25] R. R. Nair, I. L. Tsai, M. Sepioni, O. Lehtinen, J. Keinonen, A. Krasheninnikov, A. et al., Dual origin of defect magnetism in graphene and its reversible switching by molecular doping, Nat. Commun., 2013, 4, 2010.

[26] Y.-B. Zhou, L. Zhang, D.-P. Yu, Z.-M. Liao, Magnetic field induced insulating state in bilayer graphene at charge neutral point, Appl. Phys. Lett. 2014, 104153103.

[27] R. T. Weitz, M. T. Allen, B. E. Feldman, J. Martin, A. Yacoby, Broken-Symmetry States in Doubly Gated Suspended Bilayer Graphene, Science, 2010, 330, 812-816.

[28] Ji, S.-H.; Hannon, J. B.; Tromp, R. M.; Perebeinos, V.; Tersoff, J.; Ross, F. M., Atomic-scale transport in epitaxial graphene. Nat Mater 2012, 11, 114-119. 
[29] Giannazzo, F.; Deretzis, I.; La Magna, A.; Roccaforte, F.; Yakimova, R., Electronic transport at monolayer-bilayer junctions in epitaxial graphene on SiC. Phys.Rev. B 2012, 86, 235422.

[30] O. Leenaerts, B. Partoens, F. M. Peeters, Adsorption of $\mathrm{H}_{2} \mathrm{O}, \mathrm{NH}_{3}, \mathrm{CO}, \mathrm{NO}_{2}$, and $\mathrm{NO}$ on Graphene: A First-Principles Study, Phys. Rev. B, 2008, 77, 125416.

[31] O. Leenaerts, B. Partoens, F. M. Peeters, Paramagnetic Adsorbates on Graphene: A Charge Transfer Analysis, Appl. Phys. Lett. 2008, 92, 243125.

[32] F. M. Koehler, A. Jacobsen, K. Ensslin, C. Stampfer, W. J. Stark, Selective Chemical Modification of Graphene Surfaces: Distinction Between Single-and Bilayer Graphene, Small, 2010, 6, 1125-1130.

[33] R. Sharma, J. H. Baik, C. J. Perera, M. Strano, Anomalously large reactivity of single graphene layers and edges toward electron transfer chemistries, Nano lett, 2010, 10, 398-405. 